Fecha de recepción: diciembre 2012 Fecha de aceptación: septiembre 2013 Versión final: septiembre 2014

\section{La investigación y el diagnóstico de proyectos de diseño}

Gloria Angélica Martínez de la Peña *

Resumen: El objetivo de este documento es exponer y socializar experiencias docentes adquiridas durante la materia denominada Proyecto Terminal I, en la cual los alumnos deben ser capaces de plantear y formular un proyecto final en la licenciatura de Diseño de la Universidad Autónoma Metropolitana Unidad Cuajimalpa (UAM-C). Uno de los retos fundamentales consiste en iniciar a los alumnos en una dinámica y actitud de "diseñador-investigador". Para esto se profundiza en la enseñanza-aprendizaje de procesos teóricos, metodológicos y técnicos de investigación; tanto cualitativos como cuantitativos. Con base en estos conocimientos se orienta a los alumnos para que puedan diagnosticar problemáticas específicas de diseño y posteriormente plasmarlas en un protocolo de investigación.

Palabras clave: diseñador-investigador - Diseño - Proyecto terminal - UAM Cuajimalpa (UAM-C).

[Resúmenes en inglés y portugués en la página 131]

${ }^{(*)}$ Doctora en Ciencias y Artes para el Diseño. Profesor Investigador Invitado en la carrera de Diseño del Departamento de Teoría y Procesos del Diseño de la División de Ciencias de la Comunicación y Diseño en la Universidad Autónoma Metropolitana (UAM, Cuajimalpa).

\title{
Introducción
}

En este documento se presenta cuál es la esencia académica de la UAM Cuajimalpa con base en su estructura curricular y su modelo educativo. A partir de este antecedente, se expone la dinámica de la Unidad de Enseñanza-Aprendizaje (UEA) denominada Proyecto Terminal, en la cual los alumnos de diseño desarrollan durante un año, o tres trimestres del calendario escolar, un proyecto completo de investigación en diseño que identifica tres grandes momentos: la investigación del problema de diseño; la gestión y desarrollo de una propuesta diseñística integral y la evaluación de ésta. Además, a lo largo de estas UEAs los estudiantes desarrollan un documento escrito a modo de reporte de investigación denominado Idónea Comunicación de Resultados (ICR). 


\section{Antecedentes}

La Universidad Autónoma Metropolitana Unidad Cuajimalpa consciente de las exigencias de la sociedad hacia las instituciones de educación superior ha buscado "favorecer la construcción del saber por parte de los estudiantes, propiciando en ellos la capacidad de aprender, centrándose así en el compromiso de formar individuos con la capacidad de aprender constantemente, de aprender siempre" (Fresán Orozco 2005, p. 23 ).

Esta capacidad implica, siguiendo a la misma autora, una responsabilidad en el dominio de herramientas y lenguajes que faciliten la aproximación a las fuentes de información y la interpretación de los nuevos conocimientos, suponiendo una habilidad para buscar, discriminar y seleccionar información pertinente para los fines que se propone el individuo. Con lo anterior se desea enfatizar que para la UAM-C, resulta fundamental centrar las dinámicas educativas en la educación centrada en el aprendizaje de los alumnos, los cuales deben ser responsables de los conocimientos que adquieren, además de formarse como personas comprometidas con los procesos de investigación que este modelo exige hacia la construcción de nuevos conocimientos. Se busca entonces que los alumnos mantengan una participación activa en la investigación, y las materias de Proyecto Terminal I, II y III pretenden ser el ambiente y espacio idóneo para el desarrollo y consolidación de estas habilidades y aptitudes, que producirán diferencias cualitativas en su formación,teniendo como objetivo estimularlos a cuestionar y a ser críticos del conocimiento, con el propósito de impulsar cambios sociales.

Como se ha mencionado, la estructura curricular de la UAM-C apuntala un proceso de enseñanza-aprendizaje centrado en el estudiante y sustentado en la indagación, la búsqueda y la apropiación del conocimiento, realizado en forma compartida y corresponsable entre profesores y alumnos. También el trabajo grupal orientado hacia la solución de problemas de investigación en el campo del conocimiento (objetos de conocimiento) o en los problemas sociales y humanos (problemas) implicando la participación activa de los alumnos, el trabajo en equipo, la integración de contenidos teóricos, técnicos y metodológicos para integrar el conocimiento mediante el trabajo colectivo de los alumnos en actividades coordinadas por los profesores, favoreciendo así la habilitación de los estudiantes en el dominio de recursos para la solución de desafíos en el campo profesional: interdisciplina, trabajo en equipo y creatividad (Fresán, Outón y Rodríguez 2005, p. 33). Siendo éste el espíritu plasmado en las Políticas Operacionales de Docencia de la UAM-C, durante el desarrollo de las Unidades de Enseñanza Aprendizaje denominadas Proyecto Terminal, se persigue que éstas sean llevadas a cabo con diligencia a fin de lograr una congruencia entre la teoría y la práctica. De este modo, se busca mantener una coherencia entre las tres características fundamentales que conforman el modelo pedagógico de nuestra universidad: a) que es un modelo flexible, b) que se centra en el que aprende (aprender a aprender) y c) la búsqueda de la innovación constante. (Fresán, Outón y Rodríguez 2005, pp. 43-44). 


\section{Desarrollo}

La carrera de Diseño en la Universidad Autónoma Metropolitana Cuajimalpa tiene una duración prevista (deseable) de doce trimestres dividida en tres niveles generales: el primero, que corresponde al tronco general formación inicial, el segundo nivel que concierne a la formación básica y el tercero que se enfoca en la formación profesional. Existen además otras UEAs denominadas Optativas de orientación; UEAs divisionales e interdivisionales y otras denominadas Optativas de movilidad de intercambio.

Durante los trimestres del $10^{\circ}$ al $12^{\circ}$ en cada carrera se cursa una serie de Unidades de Enseñanza Aprendizaje (UEA) que se han denominado Proyecto Terminal. Éstas constan de tres cursos seriados: Proyecto Terminal I, Proyecto Terminal II y Proyecto Terminal III, que se ubican en el Tercer nivel del plan de estudios de la carrera de Diseño de la UAM-C denominado Formación profesional. El objetivo específico de este nivel es ampliar y consolidar las experiencias de diseño con los últimos adelantos en el terreno tecnológico, semiótico y estético, cristalizados en la dimensión de la sustentabilidad social y económica de sus productos. Los alumnos pueden incorporar a su proceso creativo, redes y vínculos multidisciplinares desde una perspectiva madura de su formación como diseñadores, es decir, en condiciones para enfrentar proyectos específicos de diseño de mediana complejidad. Específicamente en la carrera de Diseño de la UAM-C durante la UEA de Proyecto Terminal I, de acuerdo con el Programa de estudios, se busca que

el alumno sea capaz de reconocer y aplicar los conocimientos adquiridos, para el planteamiento y formulación de un proyecto terminal de investigación y desarrollo en el área de orientación profesional elegida por el mismo; además de que el alumno adquiera y aplique nuevos conocimientos teóricos, metodológicos y técnicos en la solución de problemáticas en el campo del diseño, de acuerdo con las necesidades del proyecto terminal en sus fases de planteamiento, fundamentación y formulación (UAM-C).

Es así, que de acuerdo con el programa operativo de Proyecto Terminal I,se puede observar que es una UEA de integración entre los cursos teóricos y prácticos en la formación del estudiante. En este curso se emplea a profundidad el modelo educativo de la UAM-C que se fundamenta en el aprendizaje basado en problemas; el proceso de enseñanza-aprendizaje que se sustenta en la indagación, búsqueda y apropiación del conocimiento; el cual se realiza en forma compartida y corresponsable entre profesores y alumnos siendo estos últimos los motivadores del mismo. Ya que nuestro modelo educativo se centra en los alumnos, son ellos, quienes deberán ser la parte activa y propositiva del curso, siempre con una actitud positiva hacia el auto-aprendizaje. Se requiere un trabajo dinámico donde los estudiantes participen constantemente en la adquisición de su conocimiento; estimulando el trabajo colaborativo con base en grupos pequeños así como de forma individual, donde los profesores no son más que facilitadores del aprendizaje. 
Los objetivos específicos de Proyecto terminal I en $10^{\circ}$ trimestre, son que al finalizarlo, el alumno sea capaz de llevar a cabo una investigación centrada en detectar necesidades latentes, particularmente necesidades y requerimientos delos usuarios; identificar y definir problemas; conceptualizar y articular soluciones factibles, recabar documentación, jerarquizarla y clasificarla para finalmente lograr en los dos siguientes trimestres (Proyecto Terminal II y III) la gestión, materialización y evaluación de la propuesta de diseño integral. De forma sintética, en el siguiente cuadro se determinan las actividades a desarrollar en cada una de las UEAs de Proyecto Terminal, siendo el objetivo de este documento mostrar únicamente el primer momento, correspondiente a Proyecto Terminal I.

\begin{tabular}{|c|c|c|c|}
\hline UEA & $\begin{array}{l}\text { PROYECTO } \\
\text { TERMINAL I }\end{array}$ & $\begin{array}{l}\text { PROYECTO } \\
\text { TERMINAL II }\end{array}$ & $\begin{array}{l}\text { PROYECTO } \\
\text { TERMINAL III }\end{array}$ \\
\hline $\begin{array}{l}\text { ACTIVIDADES A } \\
\text { DESARROLLAR } \\
\text { ALCANCES DE } \\
\text { LA UEA }\end{array}$ & $\begin{array}{l}\text { DIAGNÓSTICO } \\
\text { DE NECESIDADES } \\
\text { DE DISEÑO Y } \\
\text { DETERMINACIÓN } \\
\text { DEL PROBLEMA } \\
\text { DE DISEÑO A } \\
\text { INVESTIGAR Y } \\
\text { RESOLVER } \\
\text { DESARROLLO DE } \\
\text { LA INVESTIGACIÓN } \\
\text { TEÓRICA Y DE } \\
\text { CAMPO DEL } \\
\text { PROYECTO } \\
\text { TERMINAL } \\
\text { ELABORACIÓN DEL } \\
\text { PROTOCOLO DE } \\
\text { INVESTIGACIÓN } \\
\text { CONSTRUCCIÓN } \\
\text { DEL MARCO } \\
\text { TEÓRICO }\end{array}$ & $\begin{array}{l}\text { REFINAMIENTO DEL } \\
\text { PROTOCOLO DE } \\
\text { INVESTIGACIÓN } \\
\text { DESARROLLO } \\
\text { DE ESTRATEGIAS } \\
\text { INTEGRALES DE } \\
\text { DISEÑO QUE } \\
\text { SOLUCIONEN LAS } \\
\text { PROBLEMÁTICAS } \\
\text { DETECTADAS Y } \\
\text { LAS NECESIDADES } \\
\text { ESPECÍFICAS DE LOS } \\
\text { USUARIOS CON BASE } \\
\text { EN UN MÉTODO DE } \\
\text { DISEÑO }\end{array}$ & $\begin{array}{l}\text { REFINAMIENTO DE LA } \\
\text { SOLUCIÓN DE DISEÑO } \\
\text { MEDIANTE MODELOS } \\
\text { FUNCIONALES } \\
\text { (ENTREGABLES) } \\
\text { EVALUACIÓN } \\
\text { CONSTANTE DE LOS } \\
\text { DISEÑOS POR PARTE } \\
\text { DE LOS USUARIOS } \\
\text { A QUIENES VAN } \\
\text { DIRIGIDOS A FIN DE } \\
\text { LOGRAR PROPUESTAS } \\
\text { VIABLES } \\
\text { REDACCIÓN } \\
\text { DE LA IDÓNEA } \\
\text { COMUNICACIÓN DE } \\
\text { RESULTADOS }\end{array}$ \\
\hline
\end{tabular}

Figura 1. Cuadro de actividades a desarrollar en cada una de las UEAs de Proyecto Terminal. Fuente. UAM-C. 


\section{¿Qué se enseña en Proyecto Terminal I y para qué?}

De forma sintética, en este curso los alumnos deben ser capaces de:

1.- Identificar problemas y oportunidades de innovación y diseño.

2.- Seleccionar el objeto de investigación y desarrollo.

3.- Analizar las problemáticas de investigación definidas en la identificación del objetivo

4.- Recabar información utilizando al usuario como fuente principal de información, así como fuentes secundarias y opiniones de expertos y especialistas

5.- Evaluar la información recopilada con base en criterios de diseño, necesidades de usuarios, mercados, además de contextos sociales, culturales y ambientales.

6.- Formular una propuesta de diseño que de solución al problema planteado fundamentado en un proceso de análisis, síntesis y evaluación de información.

Durante esta UEA, lo más importante es que los alumnos de diseño sean capaces de abordar un objeto de estudio particular y que con base en la utilización de diferentes métodos, herramientas y técnicas de investigación logren diagnosticar una problemática de diseño específica y determinar cuáles las necesidades particulares de los usuarios que requieren ser satisfechas mediante soluciones integrales de diseño.

En esta UEA, los alumnos deben demostrar sus habilidades y conocimientos como diseñadores-investigadores. Una cuestión importante es que a los alumnos debe quedarles muy claro que investigar no es solamente acudir a la biblioteca a consultar libros y a hacer resúmenes o fichas de éstos; que tampoco es buscar lo referente al problema abordado en internet, y hacer un copiado y pegado (copy-paste) indiscriminado de información, que muchas veces ni leen ni resulta pertinente para el problema. Que leer sin analizar no sirve de mucho en el diagnóstico de problemas de diseño.

Es importante que los estudiantes de diseño comprendan que primero tienen que leer, analizar y sintetizar información; que deben examinar diferentes bibliografías, hacer búsquedas en bases de datos, revisar diferentes autores y que sean capaces de vincular el objeto de estudio abordado con otras disciplinas que mantengan relación con éste. En fin,que demuestren un involucramiento a profundidad con su objeto de estudio que les facilite diagnosticar problemas específicos, así como plantear preguntas y objetivos de investigación pertinentes.

Los alumnos deben comprender que los problemas de diseño no se encuentran aislados, sino que por el contrario, generalmente se encuentran inmersos en contextos complejos que requieren un abordaje sistematizado desde diferentes aristas, y que en éstos intervienen factores culturales, medioambientales, históricos, ideológicos, económicos, políticos, sociales, psicológicos, antropológicos y filosóficos, entre otros.

En este momento de su formación, deben aprender que para investigar se requieren muchas habilidades de análisis y síntesis de datos para que éstos se conviertan en información relevante. Que en los casos de diseño, los problemas reales que conforman proyectos específicos se encuentran cuando se realiza una investigación profunda centrada en y con las personas (usuarios). 
El papel del docente consiste en acompañar a los estudiantes para que dentro del aula se les pueda ofrecer aquellos contenidos que ellos necesiten para lograr las acciones mencionadas; además requiere un trabajo constante y cercano con los alumnos mediante asesorías y revisiones para ir observando sus avances y poder guiarlos a centrar sus hallazgos de forma pertinente. No se ha mencionado aún, pero cada una de estas UEAs de Proyecto Terminal I, II y III tiene una duración de doce horas semanales, divididas en tres sesiones de cuatro horas cada una durante once semanas por trimestre.

Algunos de los contenidos sintéticos que se revisan en la UEA de Proyecto Terminal I son: identificación de oportunidades de innovación y diseño; selección del objeto de investigación y desarrollo; planteamiento y formulación del problema de; identificación de los criterios para la selección de enfoques teóricos y metodológicos para realizar procesos de investigación y desarrollo para abordar el problema de diseño planteado; y finalmente que los estudiantes lleguen, como se ha mencionado, a la formulación de un proyecto de investigación y desarrollo para la identificación de requerimientos de diseño, usuarios, mercados y contextos meta.

Para abordar los contenidos de esta materia se han diseñado dinámicas docentes que consisten tanto en la exposición de algunos contenidos por parte de los maestros, como en la exposición de algunos otros por parte de los estudiantes, siempre procurando la discusión en clase y el debate constante de ideas en igualdad de condiciones. También se imparten conferencias de especialistas provenientes de otras disciplinas y se asiste a seminarios, principalmente de investigación. Por otra parte, los alumnos tienen completa libertad para buscar asesores externos especializados (dentro de la UAM y fuera de ella) que puedan guiarlos con base en sus áreas de especialidad, si así lo requieren los proyectos de investigación y desarrollo.

Durante las clases se revisan temas específicos del diseño que versan sobre tópicos como: cuál es el contexto del diseño en el siglo XXI, cuáles son las prioridades actuales del diseño, cómo se inserta el diseño en la cultura, cuáles son las discusiones contemporáneas del diseño en relación con la innovación, la gestión de proyectos, la investigación de los usuarios y el diseño de experiencias.

Otros contenidos importantes radican en el estudio y entendimiento social del comportamiento humano específicamente desde el diseño; otros versan sobre el estudio de estrategias de identificación de problemas; la investigación y uso de métodos etnográficos como la observación, la entrevista, el focus group, y algunos otros como las matrices de comparación, el desarrollo de criterios e indicadores, y el diseño de encuestas y cuestionarios.

De forma puntual se vinculan tanto la discusión como las dinámicas,a la investigación del diseño y la investigación de los usuarios y sus necesidades, mediante técnicas de investigación tanto cualitativas como cuantitativas. De igual modo, se busca profundizar en temas como la investigación-acción, la investigación en diseño para minimizar riesgos, las investigaciones de mercados, de estilos de vida, de tendencias, entre otros.

Un aspecto muy importante es que tanto la investigación teórica como la de campo para entender los contextos del diseño sea interdisciplinaria, apoyándonos en áreas del conocimiento como la antropología, la sociología, la economía, la psicología entre otras, tratando constantemente de incluir también aquellas otras disciplinas específicas que resulten pertinentes con los temas o problemas que se estén afrontando. 


\section{¿Cuáles han sido los métodos para abordar Proyecto Terminal I?}

En Proyecto Terminal I, los alumnos de diseño deben desarrollar al máximo sus capacidades y habilidades de diseñador-investigador, y en este proceso de investigación se enfrentan a problemáticas reales que les obligan a determinar cómo la disciplina que han escogido tiene una gran relevancia social que les exige determinar problemas específicos y la manera en la cual ellos deben estar preparados para afrontarlos y resolverlos mediante el diseño.

Obviamente este reto resulta importante no sólo para los alumnos sino también para los profesores, quienes asumen el papel de guías o asesores en este proceso. El método que se ha establecido en la carrera de Diseño de la UAM-C es que esta dinámica se realiza no de forma individual por los alumnos sino mediante la conformación de equipos de investigación con sus propios compañeros, lo que favorece un trabajo colaborativo en la construcción del conocimiento. Esta dinámica del trabajo en equipo presenta sus propias problemáticas, pues los estudiantes se ven obligados a resolver cualquier cantidad de desafíos y retos; no solamente aquéllos impuestos por el propio objeto de estudio, sino también por las problemáticas propias del trabajo colaborativo,y que no residen únicamente en dividirse el trabajo para resolver el proyecto en grupo, sino que ellos habrán de desarrollar mecanismos y dinámicas que equilibren las fortalezas y debilidades individuales que como sujetos poseen. El trabajo en equipo resulta ser una de las actividades más desafiantes en esta UEA de Proyecto Terminal I, ya que una de las reglas establecidas es que el equipo que se forma en Proyecto Terminal I deberá llegar a meta hasta Proyecto Terminal III. Como es de esperarse surgen conflictos y separaciones propias de las dinámicas humanas, pero este es uno de los objetivos más importantes, lograr que los alumnos se enfrenten a la realidad de trabajar con otras personas tal y como sucede en la vida profesional de cualquier diseñador. Que sean capaces de dialogar, conciliar, negociar y llegar a acuerdos que les permitan avanzar en el transcurso del proyecto durante un año completo.

Otra dinámica particular de Proyecto Terminal de la carrera de Diseño, consiste en que ninguno de los tres cursos se imparte por un solo profesor. En mi experiencia, he impartido y compartido Proyecto Terminal I con el Dr. en Economía Sazcha Marcelo Olivera Villarroel. Este hecho de trabajar de forma interdisciplinaria dentro el aula en la investigación del diseño con un docente proveniente de otra área diferente a la mía (que es el diseño), me parece que ha beneficiado enormemente la formación de los alumnos,también a mí me ha permitido aprender significativamente del diseño desde otra disciplina complementaria a la mía.

El proceso dinámico de las clases adquiere otro ritmo al compartir los contenidos con otro profesor, ya que las intervenciones de ambos en cada uno de los temas que se revisan y que he comentado con anterioridad, suele ser un proceso constante de enriquecimiento y crecimiento tanto para los alumnos como para los docentes que participamos en esta UEA. De igual modo los procesos de revisión de los adelantos de proyecto que van presentando los alumnos resultan sumamente favorecidos al ser atendidos por dos docentes de áreas diferentes. Las observaciones, comentarios y correcciones que ambos profesores vierten interdisciplinariamente generan cambios interesantes y significativos en los proyectos conforme éstos van avanzando. Este beneficio se mantiene presente en todas 
las actividades que se desarrollan durante el curso, incluyendo los procesos y momentos de la evaluación, donde ésta no se convierte en una actividad únicamente centrada en la calificación sino en una revisión completa de todo el proceso de enseñanza-aprendizaje, fundamentada en la observación de dos disciplinas complementarias que pueden valorar el proceso de indagación de forma integral.

\section{Momentos y formas de evaluación de Proyecto Terminal I}

La evaluación de Proyecto terminal se conforma como he mencionado, en un proceso constante durante el cual se van revisando los avances de los equipos en cada una de sus investigaciones y sobre todo se enfatiza cuáles son los aprendizajes que los alumnos van obteniendo durante el trimestre. Con esta estrategia de revisiones y asesorías, los docentes pueden formarse una idea clara de cómo los alumnos van creciendo en esta habilidad de aprender a aprender,misma que los alumnos deben ir consolidando de acuerdo con el modelo pedagógico de la UAM-C.

Los equipos con frecuencia (aproximadamente cada dos semanas) exponen ante todo el grupo cuáles van siendo sus hallazgos y aprendizajes más significativos. Esta socialización de la investigación permite que cada equipo reciba observaciones y comentarios no solamente por parte de los profesores, sino también por parte de todo el grupo. Esta dinámica complementa las asesorías que se les ofrecen a los equipos por parte de los docentes y al mismo tiempo sirve como una especie de termómetro a través del cual los otros equipos van midiendo su propio avance. Otro aspecto importante de esta "socialización" de la evaluación, es que ha permitido que los alumnos vayan encontrando puntos de convergencia entre sus propias investigaciones e incluso encuentren vinculaciones entre sus propios proyectos, lo cual amplía significativamente el proceso de investigación.

Los alumnos al finalizar el curso realizan también una autoevaluación de su propio aprendizaje y al mismo tiempo coevalúan a los miembros de su propio equipo en términos de los objetivos planteados, de la colaboración, la responsabilidad y del compromiso que mostraron durante el trimestre tanto en forma individual como colectiva.

La evaluación final de los proyectos de investigación se realiza y presenta ante un grupo de profesores. Los alumnos exponen públicamente sus investigaciones en diseño y los maestros de la UAM-C conformados en una especie de sínodo, exponen sus sugerencias, comentarios y observaciones a cada uno de los equipos de esta UEA. En algunas ocasiones se invita incluso a docentes externos a la UAM, ajenos a la dinámica docente de la universidad pero pertenecientes a las áreas de diseño, con la finalidad de que ellos expresen de igual forma cuáles son sus observaciones y comentarios respecto de las investigaciones y presentaciones de nuestros alumnos. 


\section{Hacia la construcción del reporte de investigación}

Como uno de los objetivos de estas UEAs de Proyecto terminal, es que los estudiantes generen un manuscrito en proyecto Terminal III, denominado Idónea Comunicación de Resultados (ICR). Es así que desde el inicio, en Proyecto Terminal I comienzan a redactar este documento en formato de protocolo de investigación.

Los rubros que deben contemplar y desarrollar en esta primera etapa de la generación de la ICR en formato de protocolo son:

1. Planteamiento del problema

Indica el tema, que debe estar directamente relacionado con el diseño. Debe dar idea precisa del tema del proyecto.

2. Objetivo(s) general (es)

Se plantea el resultado que se va a alcanzar en el Proyecto Terminal.

3. Objetivo(s) particular (es)

Se describen los módulos terminados que se espera obtener del proyecto, o bien, las etapas por donde pasa el desarrollo del Proyecto Terminal.

Para redactar los objetivos tanto general(es) como particulares, deben utilizarse verbos que puedan ser evaluados: elaborar, construir, diseñar, modelar, etc.

4. Antecedentes

Indicar qué otros proyectos se han desarrollado que tengan alguna relación con el proyecto que se desea elaborar. Considerar las siguientes fuentes:

Referencias internas (de la UAM): realizar comparación con proyectos reportados en la UAM.

Referencias externas (adquiridas fuera de la UAM): de tipo comercial, congresos, ponencias, publicaciones que estén relacionadas con el proyecto;

En ambos casos, mencionar la ficha completa de los autores, fecha en que se realizó, institución, asesores, etc.

Internet: páginas consultadas. Anotar la fecha de la consulta.

Experiencia previa que tienen los alumnos sobre el tema.

Indicar la relación entre los antecedentes y lo que se quiere hacer.

5. Justificación

Relevancia del problema que resuelve. ¿Por qué es importante y actual el proyecto? ¿Qué problema de diseño se resuelve? ¿Qué es lo que se va a obtener?

Méritos u originalidad de la solución.

En caso de existir algún proyecto parecido, se deberá indicar cuál será la aportación del proyecto particular a desarrollar y ¿cuál sería la posible continuación de este proyecto a futuro?

6. Preguntas de investigación

7. Marco teórico

Análisis e investigación (documental y de campo) de todos los componentes del sistema que conforman el problema complejo de diseño a investigar. (Análisis del sistema de diseño) 
8. Diagnóstico/Delimitación del problema de diseño (metodología)

Análisis e investigación contextual (involucrados directos e indirectos, objetos (mediadores de las actividades), entorno de uso y actividades).

Objetual (Funcional, expresivo, tecnológico y comercial)

Determinación de necesidades y requerimientos de los usuarios del proyecto de investigación y diseño

9. Plan de trabajo

Contendrá las actividades indicadas en los objetivos particulares. Constará de tres matrices de once semanas cada una, en las que se describan:

Las actividades en las que se subdivide el proyecto.

Un producto evaluable para cada una de las actividades.

La delimitación de las actividades para cada alumno que participa en el proyecto. Es decir, las actividades deberán separarse individualmente, para poder evaluar el trabajo de cada uno.

10. Bibliografía

\section{Algunos resultados obtenidos}

Durante el desarrollo de Proyecto Terminal en el periodo de abril de 2011 a abril de 2012, se abordó como objeto de estudio la Universidad, específicamente la nueva sede definitiva que se construye de la UAM Cuajimalpa en el Escorpión, ubicada en el área de Santa Fe, en la Ciudad de México. Durante un año, cinco equipos de alumnos trabajaron en diagnosticar áreas de oportunidad dentro de nuestra universida dque permitieran crear o mejorar algunas áreas o servicios universitarios.

Durante este periodo, los alumnos realizaron tanto una investigación teórica como de campo. Analizaron los orígenes de la Universidad como institución, se involucraron en el conocimiento profundo de la UAM e identificaron las diferentes propuestas educativas de cada una de nuestras unidades: por asignaturas en la Unidad Iztapalapa, por eslabones en la UAM Azcapotzalco y por módulos en Xochimilco. Del mismo modo, adquirieron un conocimiento más profundo de su propia Universidad y del modelo educativo que propone y la caracteriza.

Además, el haber realizado investigación de campo en las otras unidades de la UAM e incluso en otras instituciones de educación superior en el Área Metropolitana les permitió identificar con mayor pertinencia problemáticas específicas dentro de nuestra propia unidad Cuajimalpa.

Las propuestas obtenidas versaron sobre:

- Cómo el espacio construido genera diferentes percepciones y emociones. Este proyecto tuvo como objetivo el rediseño de la cafetería universitaria de acuerdo con las necesidades alimenticias de todos los usuarios universitarios: alumnos, docentes, administrativos y personal de apoyo. Además este proyecto tuvo como ejes rectores la accesibilidad para las personas con discapacidad y también la sustentabilidad, al proponer un manejo adecuado de los deshechos orgánicos de este espacio y así pudo vincularse con otro proyecto 
del mismo grupo que manejaba la recreación y el ocio.

- El equipo que propuso el diseño de áreas de ocio y recreación en la sede definitiva, apuntaba a favorecer espacios donde pudieran realizarse actividades diversas que se relacionaran con la sustentabilidad. En este caso, al aprovechar los residuos orgánicos de la cafetería universitaria, se propuso el diseño de talleres de composta, huertos verticales y manejo adecuado de deshechos. Este equipo también diseñó áreas de descanso en hamacarios, una cafetería con productos orgánicos y macrobióticos, así como terrazas al aire libre.

- Un tercer proyecto propuso la creación de un área en la universidad que denominaron "Emprende UAM". Este nuevo espacio presenta como objetivo el favorecer proyectos emprendedores de los alumnos que están por egresar, así como de ex alumnos que por cualquier razón no cuentan con el apoyo económico o con la infraestructura suficiente para desarrollarlos. Este equipo diseñó toda la estrategia de funcionamiento y operación de esta nueva dependencia universitaria. Asimismo propuso métodos operativos, organigrama funcional y el diseño integral de todos los sistemas que facilitaran el desarrollo de proyectos emprendedores e innovadores de alumnos y egresados de todas las carreras.

- Un cuarto equipo tomó como objeto específico de estudio el modelo educativo de la UAM-C centrado en "aprender a aprender", y cómo este modelo debe tener en consecuencia un diseño adecuado de aulas que favorezcan las dinámicas de autoaprendizaje y de trabajo colaborativo estos espacios. Este equipo propuso un diseño integral de las aulas que beneficiara el aprendizaje mediante herramientas que impulsaran la independencia y la autogestión del aprendizaje.

- Un último equipo presentó dos propuestas de diseño, una que responde a las necesidades de comunicación e interacción universitaria mediante el uso de la tecnología, y una segunda que se vinculaba con el equipo del aula. En el caso de la primera solución, propusieron el diseño de una interfaz que aglutinara en un solo sistema todos los medios digitales e informativos de la unidad favoreciendo así una comunicación ágil en la comunidad universitaria, el manejo de información oportuna y además el trabajo colaborativo más allá de las fronteras físicas. Asimismo diseñaron el módulo físico donde se ubicarían las pantallas táctiles que mostraran este sistema.

El otro dispositivo diseñado por este equipo que se vincula con el equipo del diseño de aulas, consiste en el diseño de pizarrones "inteligentes y digitales" de bajo costo. El sistema integral propuesto para este pizarrón fue diseñado por los alumnos, y funciona conectado a un cañón o proyector. Asimismo la información generada en este dispositivo, puede enviarse por correo electrónico a los estudiantes en formato PDF.

\section{Conclusiones}

Para terminar este documento quisiera exponer que el generar investigaciones de proyectos de diseño con los estudiantes que se encuentran en el proceso final de su formación, ha significado para los docentes de diseño de la UAM-C una serie de experiencias que nos parece importante compartir con humildad. En el Departamento de Teoría y Procesos del Diseño dentro de la División de Ciencias de la Comunicación y Diseño de la UAM C, hemos aceptado el desafío de formar de diseñadores-investigadores, lo cual creemos es 
imprescindible para nuestros estudiantes y para las necesidades actuales de nuestro país, lo que además simboliza una fortaleza importante en relación con los egresados de otras instituciones.

En la UAM-C creemos que el diseñador es en esencia un investigador, aunque en pocas ocasiones se asume como tal. Cada nuevo proyecto de diseño debe verse como un problema complejo de abordar que requiere del profesional de esta disciplina, las habilidades, aptitudes y conocimientos necesarios para afrontar las problemáticas complejas del mundo contemporáneo y también las necesidades específicas de sociedades heterogéneas. El diseñador no solo debe ser el "hacedor" de proyectos con base en pedidos específicos, sino un sujeto capaz de identificar y diagnosticar problemas en medio de una realidad caótica y que además sea capaz de proponer soluciones integrales de diseño que satisfagan las necesidades de diferentes usuarios. Al ser el diseñador de la UAM-C responsable de su aprendizaje, (como se pretende con el modelo educativo), estará siempre ávido de buscar respuestas diferentes ante las problemáticas que enfrente cuando sea un profesional.

La investigación como un proceso vivo, constante y cambiante requiere de un diseñador abierto, humilde y flexible ante los cambios que va presentando la propia investigación. Los alumnos deben vivir los procesos de investigación como un hecho real, propio de la disciplina y no es hasta que se enfrentan a este tipo de experiencias (diseñadas en Proyecto terminal), cuando ellos se asumen como agentes provocadores de cambios significativos en las vidas y experiencias de los usuarios del diseño.

Asimismo, el perfil de nuestros egresados, los vislumbra como diseñadores integrales y creativos que sean capaces de articular discursos de diseño congruentes, mediante la vinculación sistemática de diferentes lenguajes y recursos tecnológicos. Por otra parte, en la UAM-C consideramos que el diseñador debe asumirse no solo como un agente pasivo ante proyectos específicos dados por el profesor o el cliente, sino que debe ser capaz de gestionar activamente proyectos complejos de diseño con base en problemas reales y necesidades específicas de la sociedad. Esto requiere una madurez tanto en los egresados como en la planta docente que los guía, que debe asumirse y practicarse con la finalidad de responder a las exigencias de la sociedad. El hecho de que Proyecto Terminal sea una UEA que privilegie el trabajo interdisciplinario en equipos colaborativos prepara a los estudiantes para una práctica profesional acorde a los tiempos actuales que demandan profesionales preparados para este tipo de dinámicas. 


\section{Bibliografía}

Fresán Orozco, M. M. (2005). La unidad Cuajimalpa. Reeditando el comienzo de la UAM en Estructura Curricular Cuajimalpa. México: UAMC.

Fresán Orozco, M. M., Outón Lemus, M. y Rodríguez Lara, M. E. (2005). Estructura Curricular en Estructura Curricular Cuajimalpa. México: UAMC.

Plan de estudios de la carrera de Diseño de la UAM Cuajimalpa. Diponible en: http://herm es.cua.uam.mx/es/lic/diseno/plan Consultado el 13 de abril de 2012.

Cuajimalpa Va. Presentan estudiantes de Diseño sus Proyectos Terminales, una forma de acercamiento real al trabajo profesional (2012). Diponible en: www.cua.uam.mx/docs/2012 mar27-1.html Consultado el 27 abril 2012.

Summary: The purpose of this article is to present some teaching experiences acquired during the subject called Terminal Project I, in which students should be able to propose and develop a final project in the Bachelor of Design at the Universidad Autónoma Metropolitana. Unidad Cuajimalpa (UAM-C). One of the key challenges is to initiate students into a dynamic and attitude of "designer-researcher." The Subject deeps in the teaching and learning of theoretical, methodological and technical research processes, both qualitative and quantitative. Based on this knowledge students are guided to allow them to diagnose specific problems and its translation into a design research protocol.

Keywords: Design - designer-researcher - terminal project - UAM Cuajimalpa (UAM-C).

Resumo: O objetivo deste documento é expor e socializar experiências docentes adquiridas durante a matéria Projeto Terminal I, na qual os alunos devem ser capazes de formular um projeto final na licenciatura de Design da Universidade Autônoma Metropolitana Unidad Cuajimalpa (UAM-C). Uno dos retos fundamentais consiste em iniciar aos alunos numa dinâmica e atitude de "designer-pesquisador". Para lográ-lo se aprofunda no ensino-aprendizagem de processos teóricos, metodológicos e técnicos de pesquisa, tanto qualitativos como quantitativos. Com base nestes conhecimentos se orienta aos alunos para que possam diagnosticar problemáticas específicas de design para traduzi-las num protocolo de pesquisa.

Palavras chave: Design - designer-pesquisador - projeto terminal - UAM Cuajimalpa (UAM-C). 